\title{
The role of Toll-like receptor 3 and 4 in regulating the function of mesenchymal stem cells isolated from umbilical cord
}

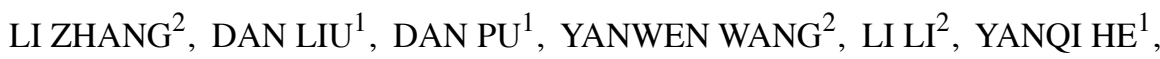 \\ YALUN LI ${ }^{1}$, LEI LI ${ }^{1}$, ZHIXIN QIU ${ }^{1}$, SHUANG ZHAO ${ }^{1}$ and WEIMIN LI ${ }^{1}$ \\ Departments of ${ }^{1}$ Respiratory Medicine, and ${ }^{2}$ Pathology, West China Hospital, \\ Sichuan University, Chengdu, Sichuan 610041, P.R. China
}

Received November 6, 2014; Accepted February 4, 2015

DOI: $10.3892 /$ ijmm.2015.2106

\begin{abstract}
Mesenchymal stem cells (MSCs) have been applied to cell-based therapy due to their multiple differentiation ability, the low expression of co-stimulatory molecules and immunosuppressive properties. Despite their immunomodulatory role, the issue of the survival and permanence of MSCs at the site of injury has not yet been fully resolved. Therefore, in order to improve the therapeutic potential of MSCs, it is important to study the mechanisms mediating the relative instability of MSCs in clinical trials. The Toll-like receptors (TLRs) are an important component of innate and adaptive immune responses. In this study, we demonstrate that the activation of two TLRs, namely TLR3 and TLR4, in human umbilical cord-derived MSCs (UCMSCs) induces the expression of inflammatory markers. In addition, as shown by our results, TLR3 upregulated the expression of stem cell markers, while TLR4 downregulated their expression. The upregulation in the expression of the inflammatory markers did not alter the immune status of the UCMSCs or mediate the immune attack of the MSCs by allogeneic immune cells. We found that the activation of TLR3 inhibited the differentiation of UCMSCs into osteocytes, while that of TLR4 increased this differentiation to a certain extent. Taken together, the results of this study provide a new role for TLR3 and TLR4 as regulators of the biological functions of UCMSCs.
\end{abstract}

\section{Introduction}

Mesenchymal stem cells (MSCs) are ubiquitous and can be found in the bone marrow, adipose tissue, skin, heart, brain, deciduous teeth, umbilical cord and peripheral blood, among others. Several characteristics of MSCs make them useful for cell-based therapies. For example, MSCs possess self-renewal capacity, multilineage differentiation potential and lack co-stim-

Correspondence to: Professor Weimin Li, Department of Respiratory Medicine, West China Hospital, Sichuan University, 37 Guo Xue Street, Chengdu, Sichuan 610041, P.R. China

E-mail:weimi003@yahoo.com

Key words: umbilical cord-derived mesenchymal stem cells, Toll-like receptor 3 and 4, polyinosinic:polycytidylic acid, lipopolysaccharide, biological functions, immunogenicity ulatory factors, such as CD80, CD86 and human leukocyte antigen (HLA); thus, they fail to activate allo- or xenogeneic immune cells due to the lack of immunogenicity (1-3). MSCs also have an immunosuppressive ability as they can inhibit the proliferation and function of T cells, B cells, natural killer (NK) cells and antigen-presenting cells (4).

The characteristic of MSCs has been applied to reduce inflammation and improve the regeneration of damaged tissue. MSCs hold great promise for the treatmetn of various diseases, such as graft versus host disease (GVHD) (5), type I diabetes (6), multiple sclerosis (7), liver cirrhosis (8) and systemic lupus erythematosus (9). Despite their promising future in cell therapy, recent clinical trials have failed to yield encouraging results. One possible explanation is that MSCs are recognized by the immune system of the recipient and are eliminated (10). In support of this notion, studies have demonstrated that MSCs express NK cell ligands that activate NK cell-mediated immune rejection (11). In addition, in murine allogeneic bone marrow transplantation, MSCs were shown to be rejected by the memory $\mathrm{T}$ cell response (12).

Toll-like receptors (TLRs) are a family of pathogenassociated molecular patterns (PAMPs), which can induce immune responses. They are activated by both components of many pathogens and endogenous ligands (13). There are 11 TLR members in human cells and they bind distinct microbial products from bacteria, viruses and fungi (14). Amongst these, TLR3 can recognize double-stranded RNA in viruses and TLR4 can bind to lipopolysaccharide (LPS), a component of Gram-negative bacteria $(15,16)$. Previous studies have demonstrated that TLR3 and TLR4 increase the osteogenic differentiation of MSCs isolated from adipose tissue and bone marrow (17). TLR3 and TLR4 have also been reported to enhance the immunosuppressive properties of human bone marrow-derived MSCS (BM-MSCs) (18).

To date, studies have focused on MSCs isolated from bone marrow and adipose tissue. Studies on MSCs isolated from the umbilical cord are limited. It has been reported that UCMSCs and BM-MSCs share high similarity in metabolic and functional processes, particularly in their immune processes and biological regulation (19). However, there are differences in the gene expression levels of neurogenesis transcription factors and growth-related genes, amongst others (20) between BM-MSCs and UCMSCs. In this study, we provide evidence of a new role 
for TLR3 and TLR4 as regulators of the biological functions of UCMSCs.

\section{Materials and methods}

Isolation and stimulation of MSCs. UCMSCs were isolated from the umbilical cord as previously described (21). Briefly, the umbilical cord was diced into sections following disinfection in $75 \%$ ethanol for 10-20 min. The small sections of mesenchymal tissues were placed into $25 \mathrm{~mm}^{2}$ plates. The culture medium was added when the tissues tightly adhered to the bottom of the plates. The dissociated MSCs were collected approximately 5-8 days after the mesenchymal tissues were removed. The dissociated MSCs were either maintained in Dulbecco's modified Eagle's medium (DMEM) supplemented with 10\% fetal bovine serum (FBS) (both from Invitrogen, Carlsbad, CA, USA) at $1 \times 10^{5}$ cells/ well in a 6-well plate (Falcon, Bedford, MA, USA) or stored in liquid nitrogen for later use. This study was approved by the Ethics Committee of West China Hospital, Chengdu, China.

The TLR3 agonist, polyinosinic:polycytidylic acid [poly(I:C); P1530], and the TLR4 agonist, LPS (L2880), were both purchased from Sigma-Aldrich (St. Louis, MO, USA) and prepared as recommended by the manufacturer.

Quantitative RT-PCR (RT-qPCR). Total RNA was isolated using an RNeasy kit (Qiagen, Dusseldorf, Germany).cDNA was synthesized using the ReverTra Ace qPCR RT kit (FSQ-101; Toyobo, Kagoshima, Japan), and the reverse transcription conditions were as follows: $65^{\circ} \mathrm{C}(5 \mathrm{~min}), 37^{\circ} \mathrm{C}(15 \mathrm{~min})$ and $98^{\circ} \mathrm{C}(5 \mathrm{~min})$. Reactions were performed using RealMaster Mix (SYBR-Green; FP202; Tiangen Biotech, Beijing, China). RT-PCR was performed in an iCycler iQ $\mathrm{i}^{\mathrm{TM}}$ Optical Module (Beckman Coulter, Fullerton, CA, USA) under the following conditions: $95^{\circ} \mathrm{C}$ for $30 \mathrm{sec}, 95^{\circ} \mathrm{C}$ for $30 \mathrm{sec}, 58^{\circ} \mathrm{C}$ for $30 \mathrm{sec}$ and $72^{\circ} \mathrm{C}$ for $30 \mathrm{sec}$, followed by a melt curve from 55 to $95^{\circ} \mathrm{C}$ in $0.5^{\circ} \mathrm{C}$ increments and $10 \mathrm{sec}$ intervals for 40 cycles. The primers used are listed in Table I. All amplifications were performed 3 times.

Antibody chip array. Supernatants were collected from the TLR3 and TLR4 agonist-treated and untreated UCMSCs at $4 \mathrm{~h}$ post-treatment and screened for secreted protein using RayBio Human Antibody Array C Series 1000 (RayBiotech, Norcross, GA, USA) according to the manufacturer's instructions.The blots were analyzed using ImageJ software (National Institutes of Health, Bethesda, MD, USA). A total of 20 molecules were selected for detection: alpha-fetoprotein (AFP), albumin, E-selectin, intercellular adhesion molecule 1 (ICAM-1), interferon (IFN)- $\alpha$, IFN- $\gamma$, interleukin (IL)-10, IL-12, IL-18, IL-1 $\beta$, IL-4, IL-5, IL-6, IL-8, monocyte chemoattractant protein (MCP)-1, MCP-3, macrophage inflammatory protein (MIP)-1 $\alpha$, Notch-1, transforming growth factor (TGF) $-\beta$ and vascular endothelial growth factor (VEGF).

Leukocyte proliferation and lactate dehydrogenase $(\mathrm{LDH})$ leakage assay. Peripheral blood leukocytes (PBLs) were isolated from healthy volunteers (after obtaining written informed consent) by density centrifugation gradient and labeled with carboxyfluorescein diacetate succinimidyl ester (CFSE) at $37^{\circ} \mathrm{C}$ for $10 \mathrm{~min}$. The reaction was terminated by the addition of ice-cold DMEM supplemented with $10 \%$ FBS, and excess the CFSE was removed with ice-cold phosphatebuffered saline (PBS). The labeled PBLs $\left(1 \times 10^{6}\right)$ were then co-cultured with the isolated UCMSCs for $72 \mathrm{~h}$ and then analyzed by FACS. All the experiments were approved by the Human Research Protection Program at our institution.

The PBLs were co-cultured with the UCMSCs and stimulated with the TLR3 and TLR4 agonists. Supernatants were then collected from the treated and untreated groups $72 \mathrm{~h}$ post co-culture and the LDH levels in the cell supernatants were measured using a cytotoxicity detection kit (Roche Applied Science, Indianapolis, IN, USA) according to the manufacturer's specifications. Cytotoxicity (percent lysis) was calculated using the following formula: $100 \times(\mathrm{E}-\mathrm{M}) /(\mathrm{T}-\mathrm{M})$, where $\mathrm{E}$ is the experimental release, $\mathrm{M}$ is the spontaneous release in the presence of medium alone, and $\mathrm{T}$ is the maximum release in the presence of 5\% Triton X-100.

Flow cytometric analysis. The TLR 3 and TLR4 agonist-treated and untreated UCSMCs were treated with either TLR-3 or TLR-4 or left untreated for $72 \mathrm{~h}$. At this point, cells were harvested and stained for the co-stimulator and surface markers listed in Table II using monoclonal antibodies. Following staining, the cells were analyzed by flow cytometry using a FACScan flow cytometer (Beckman Coulter). The cells were gated according to the fluorescence intensity of the control group.

Differentiation of UCMSCs into osteocytes. The UCMSCs were cultured in conditioned medium specific for osteocytes (A10072-01; Gibco, Carlsbad, CA, USA) and the TLR3 and TLR4 agonists according to the manufacturer's specifications. Media was replaced every 3 days and treated and untreated cells were stained after 3,10 and 14 days in culture using Alizarin red.

Western blot analysis. The TLR3 and TLR4 agonist-treated UCMSCs were washed twice with cold PBS and proteins were extracted using a standard mammalian protein extraction reagent (Pierce, Rockford, IL, USA) containing protease inhibitors (Roche Applied Science). Lysates were clarified by centrifugation at $14,000 \mathrm{rpm}$ for $10 \mathrm{~min}$ at $4^{\circ} \mathrm{C}$. The protein concentration was measured using the Micro BCA Protein Assay kit (Pierce). Protein lysates $(20 \mu \mathrm{g})$ were resolved on sodium dodecyl sulfate-polyacrylamide gels (SDS-PAGE) and transferred onto nitrocellulose membranes (Invitrogen). The blocked membranes were incubated with primary antibodies (Table III), washed and then incubated with a secondary antibody conjugated to horseradish peroxidase (1:5,000; Abcam, Cambridge, UK) for $1 \mathrm{~h}$ at room temperature. Antigen-antibody complexes were visualized using an enhanced chemiluminescence reagent (Amersham Biosciences, Fairfield, CT, USA).

Data analysis. The RT-qPCR and antibody chip data were analyzed using Bio-Rad iQ5 software (Bio-Rad, Hercules, CA, USA). Glyceraldehyde 3-phosphate dehydrogenase (GADPH) was used as an internal control. The results are expressed as the mean values \pm standard error of the mean using SPSS 16.0 software (IBM SPSS Statistics, New York, NY, USA). Values of $\mathrm{P}<0.05$ and $\mathrm{P}<0.001$ were considered to indicate statistically significant differences compared to the control group. The figures were completed using GraphPad Prism 5 software (GraphPad Software, Inc., La Jolla, CA, USA). 
Table I. Primers used for RT-qPCR.

\begin{tabular}{llll}
\hline Gene & \multicolumn{1}{c}{ Forward primer } & \multicolumn{1}{c}{ Reverse primer } & GenBank Acc.no. \\
\hline IL-1 $\beta$ & ACGAATCTCCGACCACCACT & CCATGGCCACAACAACTGAC & M15330 \\
IL-8 & CTGGCCGTGGCTCTCTTG & CCTTGGCAAAACTGCACCTT & NM_000584 \\
IL-10 & GGTGATGCCCCAAGCTGA & TCCCCCAGGGAGTTCACA & U16720 \\
IL-12 & CGGTCATCTGCCGCAAA & CAAGATGAGCTATAGTAGCGGTCCT & M65272 \\
IFN- $\beta$ & CAGCAATTTCAGTGTCAGAAGCT & TCATCCTGTCCTTGAGGCAGT & M28622 \\
TGF- $\beta$ & TATCGACATGGAGCTGGTGAAG & CAGCTTGGACAGGATCTGGC & X02812 \\
IP-10 & TGAAATTATTCCTGCAAGCCAA & CAGACATCTCTTCTCACCCTTCTTT & NM_001565 \\
CCL1 & TGCAGATCATCACCACAGCC & GTCCACATCTTCCGGCCA & NM_002981 \\
CCL2 & CTCTGCCGCCCTTCTGTG & TGCATCTGGCTGAGCGAG & NM_002982 \\
CCL4 & GACACCACACCCTGCTGCT & TACTCCTTGATGTGGGCACG & NM_005408 \\
CCL13 & CAGTGCTTCTGTGCCTGCTG & TGCATCTGGCTGAGCAAGTC & NM_004235.4 \\
Klf4 & GTCATCAGCGTCAGCAAAGG & CCCTGCTGCTCAGCACTT & NM_002448.3 \\
MSX & GGTTTCCTCTCCCTCTCCAC & AATTTGAATTTTACGTCTGCTG & NM_00129769 \\
Nanog & CCAAAGGCAAACAACCCACTT & CGGGACCTTGTCTTCCTTTTT & NM_003106.3 \\
Sox2 & CCCCTTTATTTTCCGTAGTTGTATTT & GATTCTCGGCAGACTGATTCAA & AM393287 \\
CDC2 & CAGGTTATATCTCATCTTTGAG & GTTGAGTAACGAGCTGACCCC & J04038 \\
GAPDH & GAAGGTGAAGGTCGGAGTC & GAAGATGGTGATGGGATTTC & \\
\hline PAR & & &
\end{tabular}

PCR, polymerase chain reaction; IL, interleukin; IFN- $\beta$, interferon- $\beta$; TGF- $\beta$, transforming growth factor- $\beta$; Klf4, Krüppel-like factor 4 ; MSX, muscle segment homeobox; GAPDH, glyceraldehyde 3-phosphate dehydrogenase.

Table II. Monoclonal antibodies used in FACS analysis.

\begin{tabular}{lcc}
\hline Name & Company & Catalog no. \\
\hline CD80 & eBioscience, San Diego, CA, USA & $11-0809$ \\
CD86 & eBioscience, San Diego, CA, USA & $12-0869$ \\
CD74 & eBioscience, San Diego, CA, USA & $11-0748$ \\
CD105 & eBioscience, San Diego, CA, USA & $12-1057$ \\
\hline
\end{tabular}

\section{Results}

TLR3 and TLR4 agonists increase the expression of proinflammatory cytokines, but have a different effect on the stemness of UCMSCs. The UCMSCs were treated with the TLR3 agonist, poly(I:C), and the TLR4, agonist LPS, and the expression of pro-inflammatory cytokines [IFN- $\beta$, TGF- $\beta$, IP-10 and CDC2], chemokines (CCL1, CCL2, CCL4 and CCL13) and interleukins (IL-1 $\beta$, IL-8, IL-10 and IL-12) was examined at 4, 12, 24, 72 and $12 \mathrm{~h}$ following treatment with the ligands. We found that treatment with TLR3 agonist induced the expression of IFN- $\beta$, TGF- $\beta$, CCL1, CCL4, IL-10 and IL-12 to varying extents different kinetics as shown in Fig. 1A-C. In addition, treatment with the TLR 3 agonist inhibited the expression of CDC2, CCL2 and IL- 8 at different time points. Lastly, treatment with the TLR3 agonist induced the expression of IP-10 and IL-1 $\beta$, but the expression levels decreased at later time points as shown in Fig. 1A-C.

By contrast, treatment with the TLR4 agonist downregulated the expression of CDC2 and IFN- $\beta$. However, the expression of TGF- $\beta$, IL-1 $\beta$, IL-8, IL-10 and IL-12 increased significantly at
Table III. Antibodies used for detection in western blot analysis.

\begin{tabular}{lll}
\hline Antibody name & \multicolumn{1}{c}{ Company } & Catalog no. \\
\hline GAPDH & Abcam, Cambridge, UK & Ab181602 \\
$\beta$-catennin & Abcam, Cambridge, UK & Ab32572 \\
IRAK4 & Abcam, Cambridge, UK & Ab119942 \\
ERK & Abcam, Cambridge, UK & Ab17942 \\
p38 & Abcam, Cambridge, UK & Ab7952 \\
p-STAT3 & Abcam, Cambridge, UK & Ab76315
\end{tabular}

GAPDH, glyceraldehyde phosphate dehydrogenase; IRAK4: interleukin-1 receptor-associated kinase; ERK: extracellular signal-regulated kinase; pSTAT3: phosphorylated signal transducer and activator of transcription 3 .

different time points post-treatment (4, 12, 24, 72 and $120 \mathrm{~h})$. Other markers, such as IP-10, CCL1, CCL2, CCL4 and CCL13 showed a slight variation in their expression levels following treatment with LPS (TLR4 agonist; Fig. 1A-C).

Stem cell marker expression was measured to confirm whether treatment with poly(I:C) or LPS influences the stemness of UCMSCs. We found that the expression of stem cell markers [Krüppel-like factor 4 (Klf4), muscle segment homeobox (MSX), Nanog and Sox2] increased following treatment with poly(I:C). In addition, treatment with LPS treatment markedly inhibited the expression of Klf4, MSX and Nanog, but increased the expression of Sox2 (Fig. 1D). Therefore, the activation of TLR3 and TLR4 induced the expression of proinflammatory molecules and TLR3 increased the stemness of UCMSCs, but TLR4 inhibited it. 
TLR3
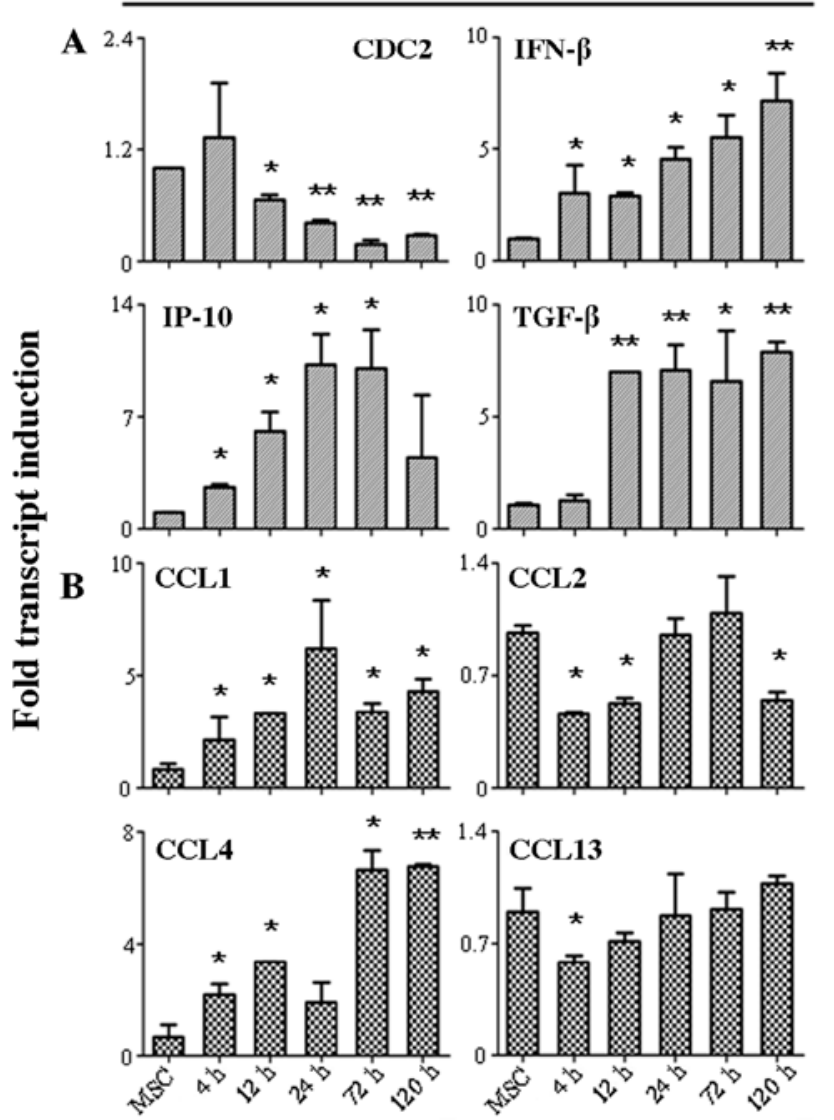

TLR4
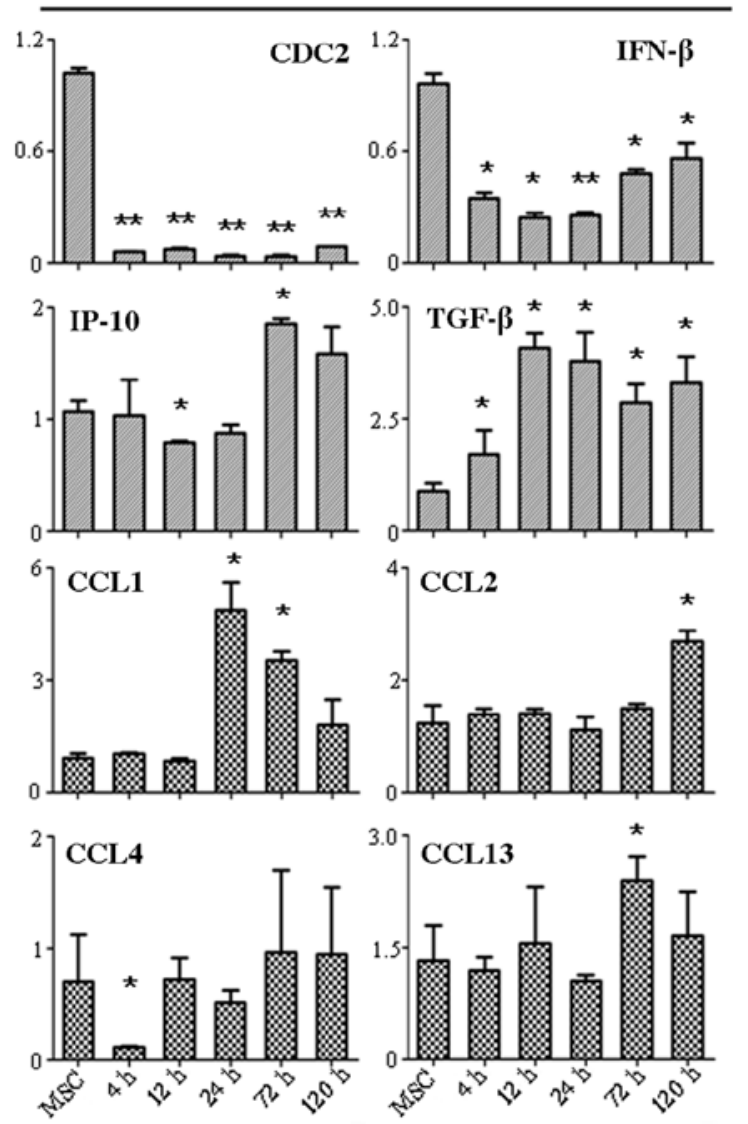
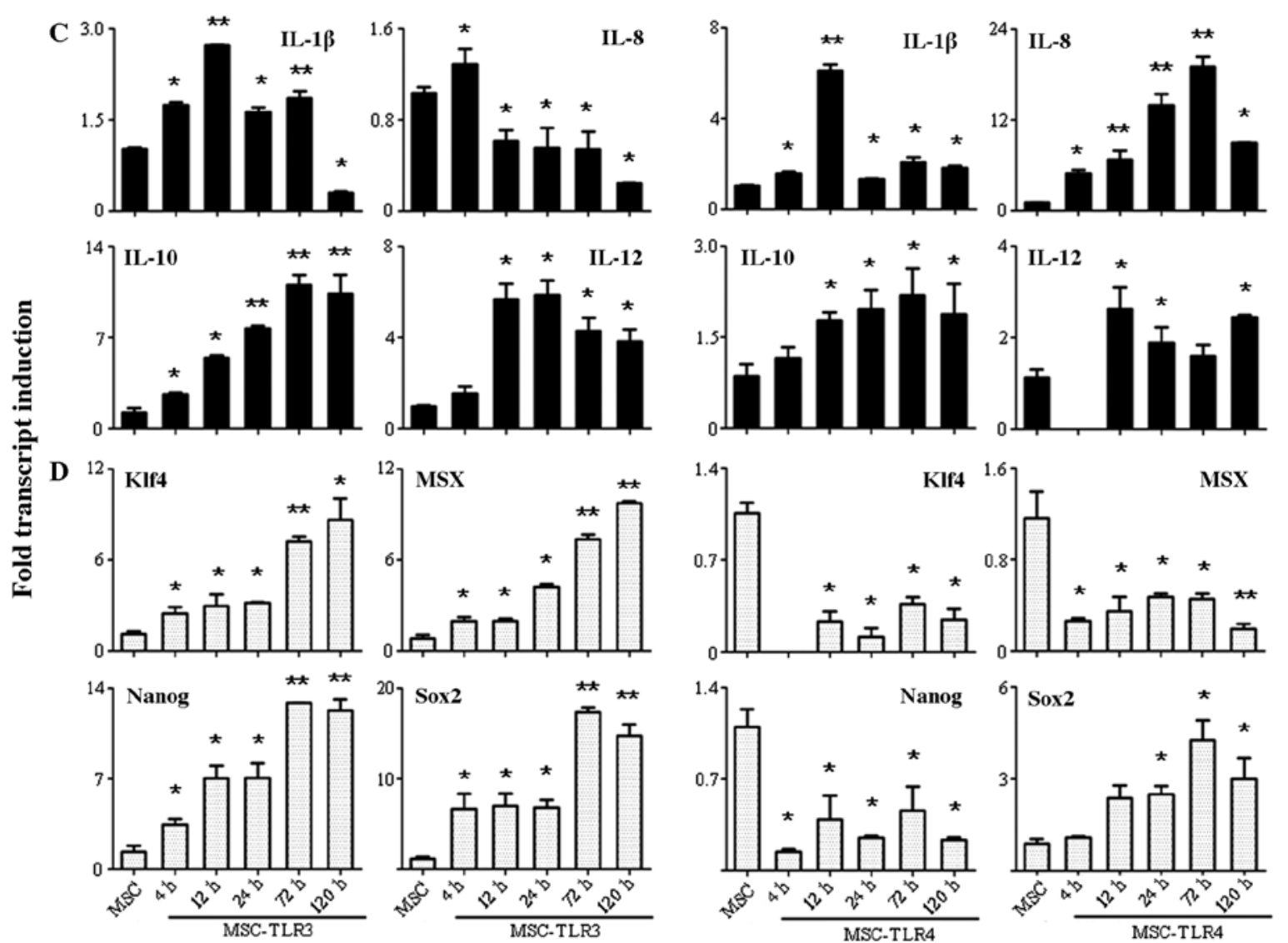

Figure 1. Toll-like receptor (TLR)3 and TLR4 agonists upregulate gene expression in umbilical cord-derived mesenchymal stem cells (UCMSCs). (A) Cytokines; (B) chemokines; (C) interleukins; (D) stem cell markers. Data are the means \pm SD from 3 independent experiments. ${ }^{* *} \mathrm{P}<0.001$ and ${ }^{*} \mathrm{P}<0.05$ vs. the control group. 


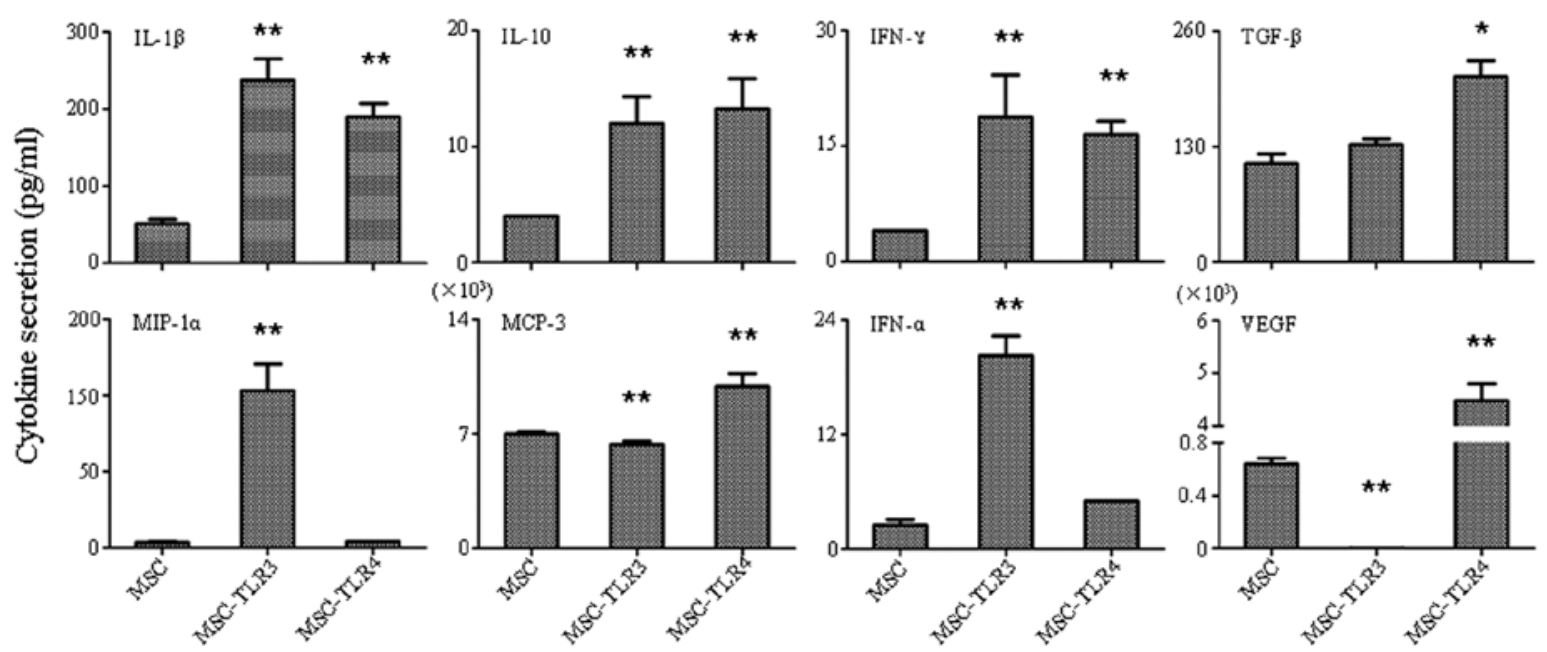

Figure 2. Activation of toll-like receptor (TLR)3 and TLR4 leads to cytokine/chemokine secretion. Cytokine/chemokine levels in the culture medium were measured as described in the Materials and methods. ${ }^{* *} \mathrm{P}<0.001$ and ${ }^{*} \mathrm{P}<0.05$ vs. the control group.
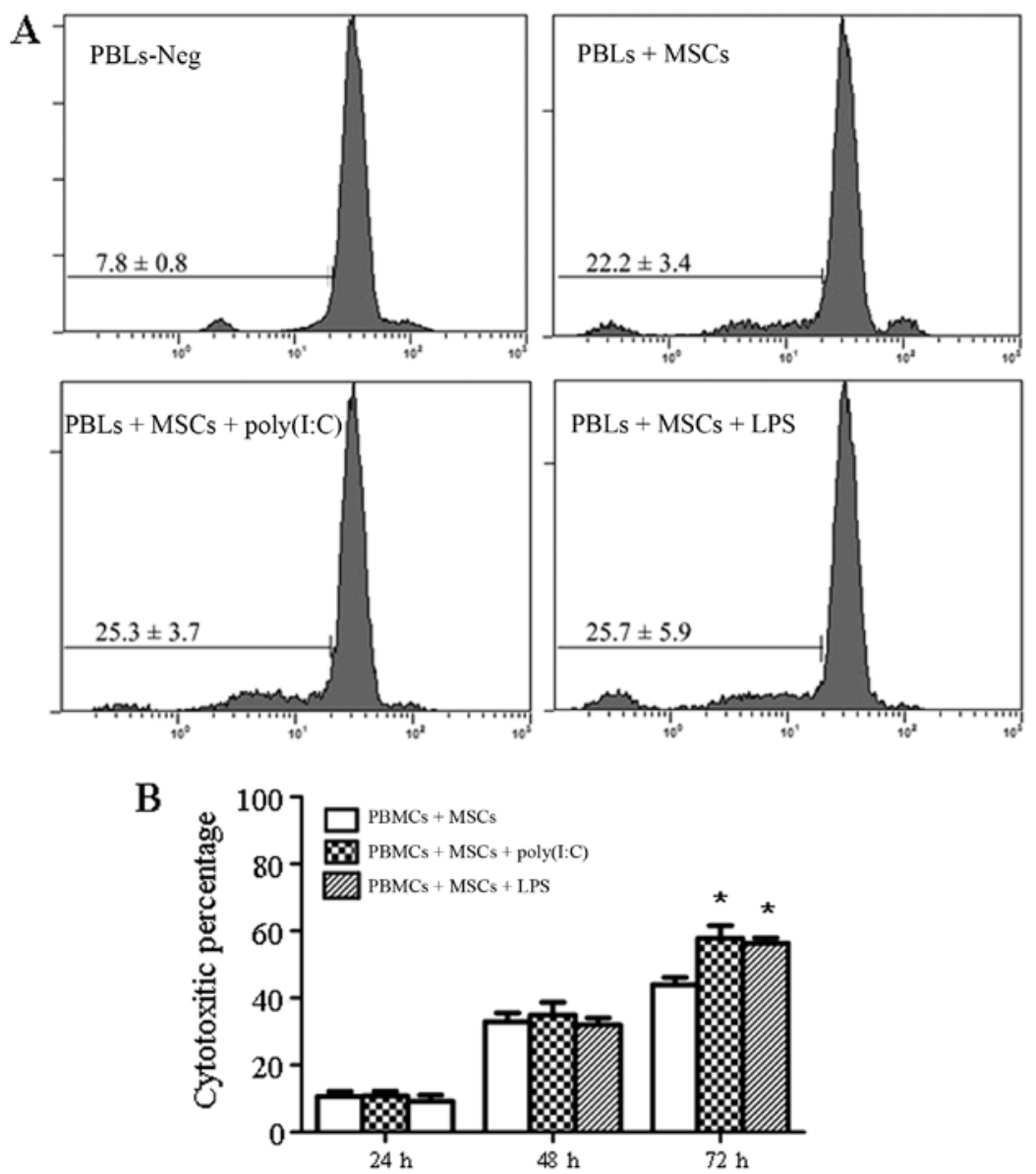

Figure 3. Toll-like receptors (TLR)3 and TLR4 agonists do not increase the proliferation of allogeneic peripheral blood leukocytes (PBLs) in response to umbilical cord-derived mesenchymal stem cells (UCMSCs). (A) Co-culture of PBLs and UCMSCs, (B) lactade dehydrogenase (LDH) detection for cytotoxicity. ${ }^{*} \mathrm{P}<0.05$ vs. the control group.

TLR3 and TLR4 ligands increase the secretion of proinflammatory molecules. Immune-related molecules in the supernatants of the treated UCMSCs were measured using the RayBio antibody chip. We then observed increased levels of IL-1 $\beta$, IL-10 and IFN- $\gamma$ in the supernatants of the UCMSCs treated with poly(I:C) or LPS (Fig. 2). The levels of TGF- $\beta$,
MCP-3 and VEGF were increased only following treatment with LPS. In addition, the levels of MIP-1 $\alpha$ and IFN- $\alpha$ were increased only following treatment with poly(I:C) (Fig. 2). Together, these results confirm that the activation TLR3 and TLR4 induces the secretion of pro-inflammatory molecules in UCMSCs. 

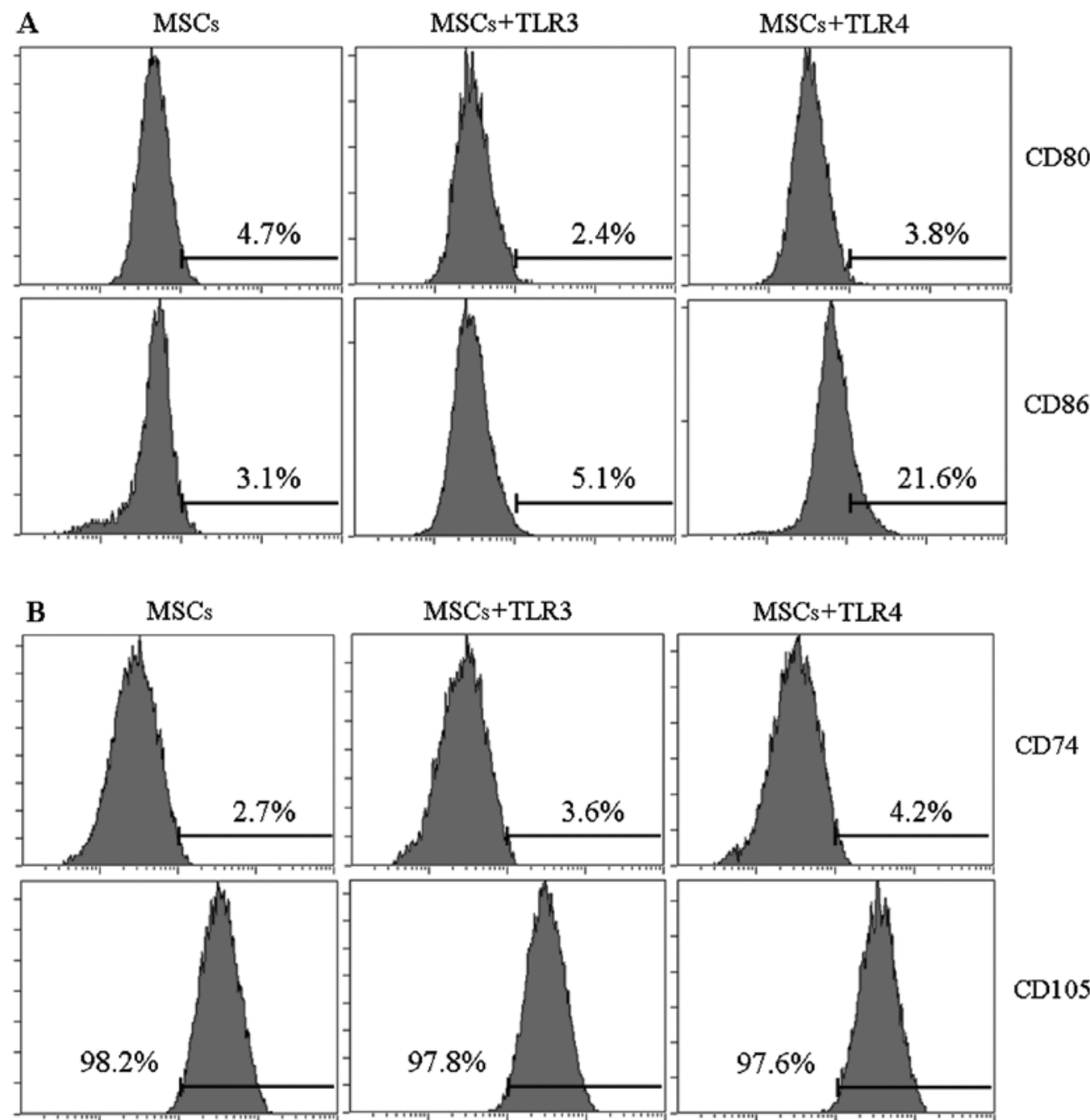

CD74

CD105

$98.2 \%$

$97.8 \%$

Figure 4. The Toll-like receptor 3 (TLR3) agonist did not upregulate the expression of co-stimulatory molecules in umbilical cord-derived mesenchymal stem cells (UCMSCs). Expression of co-stimulatory molecules and markers in MSCs. (A) Co-stimulatory molecules, (B) surface markers.

TLR3 and TLR4 agonists do not increase the proliferation of allogeneic PBLs in response to UCMSCs. The majority of the MSC-related clinical trials have been based on the assumption that MSCs can inhibit the immune response. In order to determine whether the immune suppressive function of MSCs can be compromised following treatment with TLR3 and TLR4, CFSE-labeled allogeneic human PBLs were co-cultured with UCMSCs treated or not with TLR3 and TLR4 ligands. The results revealed that the proliferation of the PBLs was not significantly affected following incubation with either the TLR3 or TLR4 agonist (Fig. 3A).

At this point, we wished to determine whether PBLs exert an immune attack against UCMSCs by measuring the levels of LDH in the cell supernatants from the co-culture of PBLs and UCMSCs. We found that the LDH levels remained relatively unaltered in both the poly(I:C)- and the LPS-treated groups at both 24 and $48 \mathrm{~h}$ post-treatment (Fig. 3B). However, the activation of TLR3 and TLR4 induced the leakage of LDH in the PBL-UCMSC co-cultures compared to the control at $72 \mathrm{~h}$ (Fig. 3B). Therefore, the activation of the TLR3 and TLR4 pathways did not promote the rejection of UCMSCs through the TLR3 and TLR4 dependent pathway.
The TLR3 agonist does not upregulate the expression of co-stimulatory molecules in UCMSCs. The data from our experiments suggested that the TLR3 and TLR4 agonists did not induce immune rejection of the UCMSCs, although they induced the expression of pro-inflammatory molecules. Therefore, we then assessed whether the activation of TLR3 and TLR4 upregulates the expression of co-stimulatory molecules, which play an important role in immune responses. We found that 2 major co-stimulatory proteins, CD80 and CD86, were unaffected following treatment with the TLR3 agonist (Fig. 4A). However, the TLR4 ligand increased the expression of CD86 (21.6\%) compared to the control (3.1\%), but did not affect CD80 expression (Fig. 4A). Similarly, the levels of the MSC-specific surface markers, CD74 and CD105, in the UCMSC following the activation of TLR3 and TLR4 were unaffected (Fig. 4B).

TLR3 and TLR4 exert opposite effects on the differentiation of UCMSCs into osteoblasts. Since TLR3 increased the expression of stem cell markers, but TLR4 inhibited them, we hypothesized that TLR3 and TLR4 play a role in the differentiation of UCMSCs. Poly(I:C) and LPS were added 
7 days
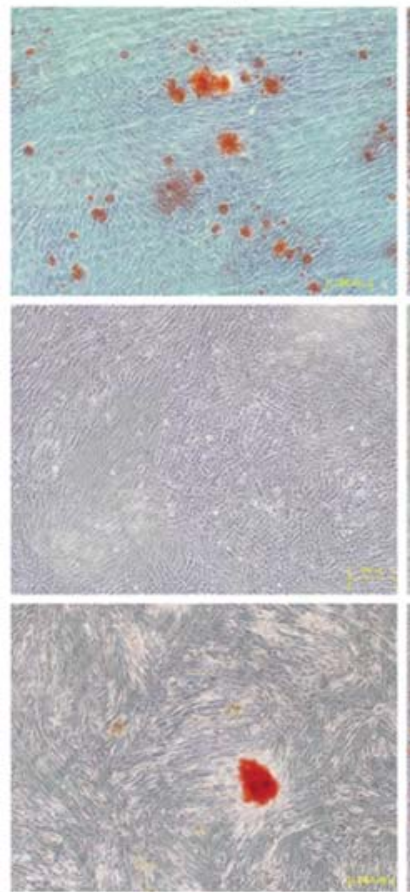

14 days
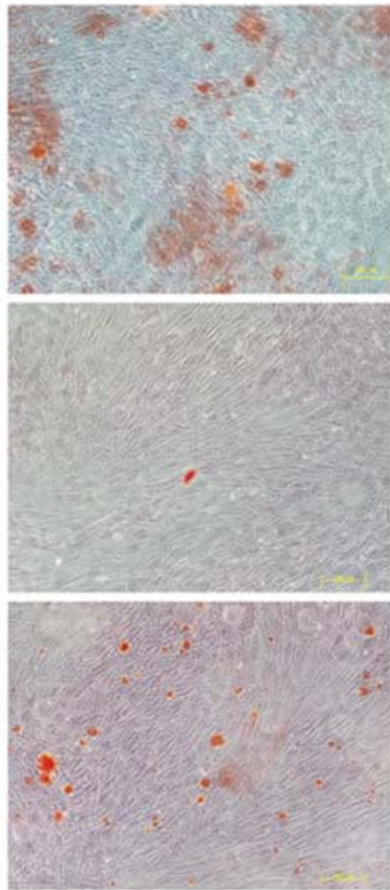

21 days

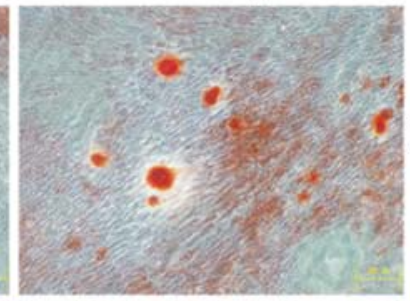

MSCs-control

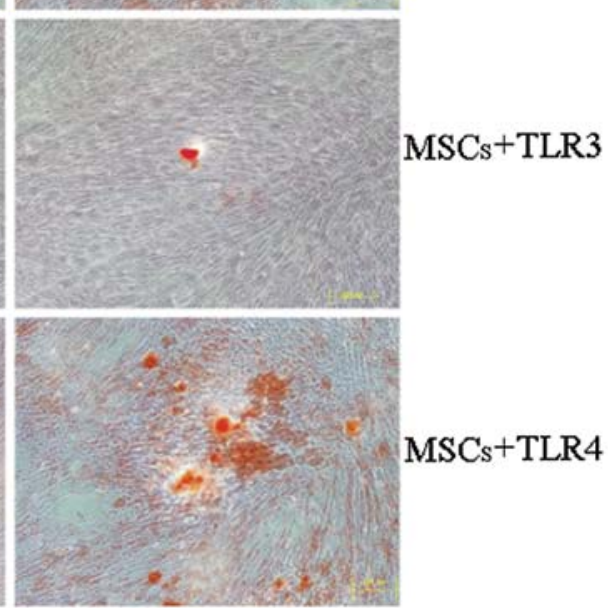

Figure 5. The activation of Toll-like receptor (TLR)3 and TLR4 has opposite effects on the differentiation of umbilical cord-derived mesenchymal stem cells (UCMSCs) into osteoblasts. Osteocyte differentiation was measured by Alizarin red staining following the activation of TLR3 and TLR4.

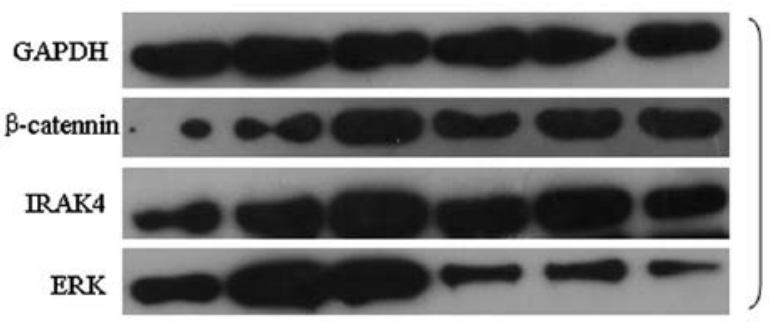

TLR3

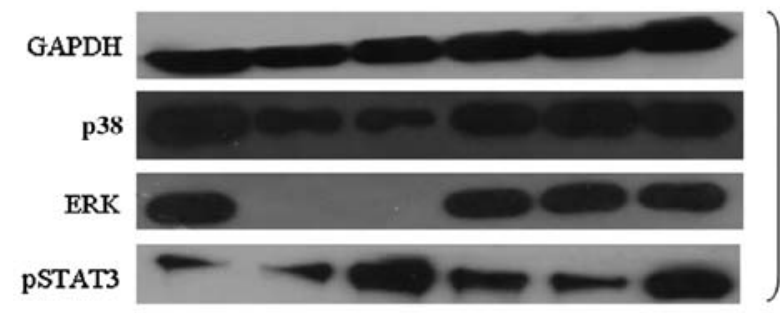

Figure 6. Activation of Toll-like receptor (TLR) 3 and TLR4 increases the expression of kinase signaling proteins. Western blot analysis for kinase signaling pathways of umbilical cord-derived mesenchymal stem cells (UCMSCs) in the presence of either the TLR3 or TLR4 agonist

to the conditioned medium and osteocyte differentiation was measured by Alizarin red staining. We found that the TLR3 agonist markedly inhibited the differentiation of UCMSCs into osteocytes, as indicated by the calcium deposition (Fig. 5). By contrast, the TLR4 agonist promoted the differentiation of USMSCs into osteocytes (Fig. 5).

Activation of TLR3 and TLR4 increases the expression of kinase signal proteins. To elucidate the signaling pathways that are activated by the TLR 3 and TLR 4 agonists, we probed for signaling pathway proteins in the UCMSCs following treatment with poly(I:C) and LPS. We found that treatment with the TLR3 agonist markedly increased $\beta$-catenin and interleukin-1 receptor-associated kinase 4 (IRAK4) levels in the UCMSCs. By contrast, the activation of TLR4 by LPS activated the phosphorylated signal transducer and activator of transcription 3 (pSTAT3) and p38 pathways (Fig. 6).

\section{Discussion}

MSCs have been applied to cell-based therapies due to their self-renewal and differentiation properties. Therefore, knowledge of the factors and mechanisms regulating these properties and their potential immunogenicity is critical for the therapeutic use of MSCs (22). Previous studies have confirmed that MSCs alter the immune status, leading to cell-based therapy failure through different mechanisms (23).

TLRs play a role in the induction of the innate and adaptive immune responses and can also activate the MSC compartment. Previous studies have demonstrated that the TLR3 and TLR4 ligands [poly(I:C) and LPS, respectively] induced the expression of inflammatory mediators, such as IL-1, IL-6, IL-8 and CCL5 and induced the recruitment of innate immune cells in vivo (24). However, these studies used BM-MSCs and not UCMSCs, which have received a lot of attention in recent clinical trials (25). In this study, we examined the role of the TLR3 and TLR4 pathways in modulating the immune properties of UCMSCs. In contrast to previously published data (26), we found that the activation of TLR3 had no effect on the immunogenicity of UCMSCs, although the expression of pro-inflammatory molecules, such as IL-1 $\beta$, IP-10, IL-10 and IL-12 was induced upon treatment with poly(I:C). In support 
of this evidence, we found that the activation of TLR3 in the UCMSCs failed to increase the proliferation of PBLs, the immune attack by allogeneic human immune cells and the expression of the co-stimulatory molecules, CD80 and CD86. Similar results were observed following the activation of TLR4 in the UCMSCs except for the slight increase in the expression of CD86 following treatment with the TLR4 agonist, LPS.

The differentiated MSCs promoted immune rejection by the host. The observed increase in the expression of stem cell markers (Klf4, MSX, Nanog and Sox2) in the TLR3-treated UCMSCs suggested that TLR3 mediates the stemness maintenance of UCMSCs. In support of this notion, we found that treatment with poly(I:C) inhibited the differentiation of UCMSCs into osteocytes. By contrast, we found that the activation of TLR4 in the UCMSCs inhibited the expression of stem cell markers (Klf4, MSX and Nanog) and partly increased the differentiation ability of the UCMSCs into osteocytes. Taken together, our data provide new evidence of the role of the TLR3 and TLR4 pathways in the regulation of the biological functions of UCMSCs.

In clinical trials, the engrafted UCMSCs can be exposed to TLR ligands, resulting in the activation of the receptors. In addition, a number of endogenous molecules, such as the heat shock proteins (HSP)60, -70 , heparin sulfate, fibronectin extra domain A, hyaluronan, oxidized LDL, uric acid, myeloid-related proteins 8 and 14, intracellular components of fragmented cells can also activate TLRs (26). These endogenous TLR ligands may regulate UCMSC function through endogenous stimuli during tissue repair at sites of tissue injury and cell necrosis. We found that TLR 3 and TLR4 induced the expression of inflammatory molecules, but did not alter the immunogenicity of UCMSCs. Future studies are required to confirm the role of the induction of inflammatory molecules in the regulation of the biological function of UCMSCs.

\section{Acknowledgements}

This study was supported by a grant from the National Natural Scientific Foundation of China (81372504).

\section{References}

1. Phinney DG and Prockop DJ: Concise review: mesenchymal stem/multipotent stromal cells: the state of transdifferentiation and modes of tissue repair - current views. Stem Cells 25: 2896-2902, 2007.

2. van Poll D, Parekkadan B, Cho CH, Berthiaume F, Nahmias Y, Tilles AW and Yarmush ML: Mesenchymal stem cell-derived molecules directly modulate hepatocellular death and regeneration in vitro and in vivo. Hepatology 47: 1634-1643, 2008.

3. Bassi EJ, Aita CA and Câmara NO: Immune regulatory properties of multipotent mesenchymal stromal cells: Where do we stand? World J Stem Cells 3: 1-8, 2011.

4. Han KH, Ro H, Hong JH, Lee EM, Cho B, Yeom HJ, Kim MG, $\mathrm{Oh} \mathrm{KH}$, Ahn $\mathrm{C}$ and Yang J: Immunosuppressive mechanisms of embryonic stem cells and mesenchymal stem cells in alloimmune response. Transpl Immunol 25: 7-15, 2011.

5. Le Blanc K, Frassoni F, Ball L, et al; Developmental Committee of the European Group for Blood and Marrow Transplantation: Mesenchymal stem cells for treatment of steroid-resistant, severe, acute graft-versus-host disease: A phase II study. Lancet 371: 1579-1586, 2008.

6. Vojtassák J, Danisovic L, Kubes M, Bakos D, Jarábek L, Ulicná $\mathrm{M}$ and Blasko M: Autologous biograft and mesenchymal stem cells in treatment of the diabetic foot. Neuro Endocrinol Lett 27 (Suppl 2): 134-137, 2006.
7. Guiducci S, Porta F, Saccardi R, et al: Autologous mesenchymal stem cells foster revascularization of ischemic limbs in systemic sclerosis: A case report. Ann Intern Med 153: 650-654, 2010.

8. Kharaziha P, Hellström PM, Noorinayer B, et al: Improvement of liver function in liver cirrhosis patients after autologous mesenchymal stem cell injection: A phase I-II clinical trial. Eur J Gastroenterol Hepatol 21: 1199-1205, 2009.

9. Sun L, Wang D, Liang J, et al: Umbilical cord mesenchymal stem cell transplantation in severe and refractory systemic lupus erythematosus. Arthritis Rheum 62: 2467-2475, 2010.

10. Allison M: Genzyme backs Osiris, despite Prochymal flop. Nat Biotechnol 27: 966-967, 2009.

11. Spaggiari GM, Capobianco A, Becchetti S, Mingari MC and Moretta L: Mesenchymal stem cell-natural killer cell interactions: Evidence that activated NK cells are capable of killing MSCs, whereas MSCs can inhibit IL-2-induced NK-cell proliferation. Blood 107: 1484-1490, 2006.

12. Nauta AJ, Westerhuis G, Kruisselbrink AB, Lurvink EG, Willemze R and Fibbe WE: Donor-derived mesenchymal stem cells are immunogenic in an allogeneic host and stimulate donor graft rejection in a nonmyeloablative setting. Blood 108: 2114-2120, 2006.

13. Akira S, Uematsu $\mathrm{S}$ and Takeuchi O: Pathogen recognition and innate immunity. Cell 124: 783-801, 2006.

14. Blasius AL and Beutler B: Intracellular Toll-like receptors. Immunity 32: 305-315, 2010.

15. Wang T, Town T, Alexopoulou L, Anderson JF, Fikrig E and Flavell RA: Toll-like receptor 3 mediates West Nile virus entry into the brain causing lethal encephalitis. Nat Med 10: 1366-1373, 2004.

16. Ghosh TK, Mickelson DJ, Solberg JC, Lipson KE, Inglefield JR and Alkan SS: TLR-TLR cross talk in human PBMC resulting in synergistic and antagonistic regulation of type- 1 and 2 interferons, IL-12 and TNF- $\alpha$. Int Immunopharmacol 7: 1111-1121, 2007.

17. Lombardo E, DelaRosa O, Mancheño-Corvo P, Menta R, Ramírez C and Büscher D: Toll-like receptor-mediated signaling in human adipose-derived stem cells: Implications for immunogenicity and immunosuppressive potential. Tissue Eng Part A 15: 1579-1589, 2009.

18. Opitz CA, Litzenburger UM, Lutz C, et al: Toll-like receptor engagement enhances the immunosuppressive properties of human bone marrow-derived mesenchymal stem cells by inducing indoleamine-2,3-dioxygenase-1 via interferon-beta and protein kinase R. Stem Cells 27: 909-919, 2009.

19. Miranda HC, Herai RH, Thomé CH, Gomes GG, Panepucci RA, Orellana MD, Covas DT, Muotri AR, Greene LJ and Faça VM: A quantitative proteomic and transcriptomic comparison of human mesenchymal stem cells from bone marrow and umbilical cord vein. Proteomics 12: 2607-2617, 2012.

20. Hsieh JY, Wang HW, Chang SJ, Liao KH, Lee IH, Lin WS, Wu CH, Lin WY and Cheng SM: Mesenchymal stem cells from human umbilical cord express preferentially secreted factors related to neuroprotection, neurogenesis, and angiogenesis. PLoS One 8: e72604, 2013.

21. Fu YS, Cheng YC, Lin MY, Cheng H, Chu PM, Chou SC, Shih YH, Ko MH and Sung MS: Conversion of human umbilical cord mesenchymal stem cells in Wharton's jelly to dopaminergic neurons in vitro: Potential therapeutic application for Parkinsonism. Stem Cells 24: 115-124, 2006.

22. Shi M, Liu ZW and Wang FS: Immunomodulatory properties and therapeutic application of mesenchymal stem cells. Clin Exp Immunol 164: 1-8, 2011.

23. Li Y and Lin F: Mesenchymal stem cells are injured by complement after their contact with serum. Blood 120: 3436-3443, 2012.

24. Tomchuck SL, Zwezdaryk KJ, Coffelt SB, Waterman RS, Danka ES and Scandurro AB: Toll-like receptors on human mesenchymal stem cells drive their migration and immunomodulating responses. Stem Cells 26: 99-107, 2008.

25. Huang YC, Parolini O, La Rocca G and Deng L: Umbilical cod versus bone marrow-derived mesenchymal stromal cells. Stem Cells Dev 21: 2900-2903, 2012.

26. DelaRosa O and Lombardo E: Modulation of adult mesenchymal stem cells activity by Toll-like receptors: Implications on therapeutic potential. Mediators Inflamm 2010: 865601, 2010. 